\title{
The relationship between the number of presented stimuli and prospective duration estimates: The effect of concurrent task activity
}

\author{
JOHN PREDEBON \\ University of Sydney, Sydney, New South Wales, Australia
}

\begin{abstract}
The attention/distraction models of prospective time estimation predict either a negative relationship or an independence between duration estimates and the number of stimulus events presented during the time period, but not a positive relationship. Two experiments examined this relationship under prospective conditions. Lists of words were presented during a 60 -sec time period at either a fast or a slow rate. Subjects either passively viewed the words or actively responded by performing either a graphemic or a semantic classification task on each word. To measure subjective duration, the method of magnitude estimation was used in Experiment 1 and the method of reproduction was used in Experiment 2. Time estimates were independent of the number of presented words in the passive viewing condition, and negatively related in the classification task conditions. Furthermore, the two classification tasks had similar effects on time estimates. These findings are consistent with the attentional models of prospective timing, and they question the robustness of earlier findings of a positive relationship between time estimates of moderately long intervals and the number of presented stimuli under conditions of minimal processing of the interval events.
\end{abstract}

Prospective timing occurs when subjects are forewarned that they will be required to estimate the duration of an upcoming time period. Under such conditions, it is frequently found that subjective duration is negatively related to the processing demands of nontemporal activities performed during the time period (for reviews, see Hicks, Miller, \& Kinsbourne, 1976; Predebon, in press; Zakay, 1993). This negative relationship is often explained in terms of the limited-capacity attention/distraction models of temporal processing (see, e.g., Thomas \& Weaver, 1975). According to these models, attentional resources are shared between a temporal processor, or timer, which counts and stores subjective temporal units (STUs), and other nontemporal processing activities. Since attention to time-in-passing is necessary for the timer's operation, diverting attention away from the timer causes fewer STUs to be created and stored during a given time interval, and, as a result, subjective duration decreases with increases in the attentional demands of the nontemporal task.

The attentional models also account for the negative relationship between the number of stimulus events presented during the time period (hereafter also referred to as stimulus quantity) and subjective duration that has been reported (McClain, 1983; Predebon, in press; Vroon, 1970 ) in situations requiring active responding to the interval events (e.g., by studying the events for an upcoming

This work was supported by an Australian Research Council grantInstitutional Grants Scheme. Correspondence may be addressed to the author at the Department of Psychology, University of Sydney, Sydney, NSW 2006, Australia (e-mail: john@psychvax.psych.su.oz.au). memory test). Increasing the number of presented stimuli increasingly diverts attention away from time-in-passing, resulting in a negative relationship between subjective duration and stimulus quantity. Of principal interest to the present investigation is the form of the relationship when subjects are not required to actively respond to the events, that is, under conditions of "passive processing" or "mere stimulus presentation" (Hicks et al., 1976). In this case, the models predict that subjective duration will be either unaffected by the interval events or negatively related to stimulus quantity if attention is inadvertently directed away from monitoring time-in-passing to the events themselves Thus, these models predict either an independence (passive processing) or a negative relationship (active processing) but exclude the possibility of a positive relationship.

In contrast, Hicks's model (Hicks, Miller, Gaes, \& Bierman, 1977; Hicks et al., 1976) of prospective timing allows for both negative and positive relationships between stimulus quantity and subjective duration, depending on the nature of the concurrent nontemporal activity performed during the time period. The model explains the negative relationship under conditions of active processing of the interval events in the same way as the attention/ distraction models. In the case of passive processing of the interval events, the model assumes that increasing the number of presented stimuli increases the number of STUs created and stored during the interval, resulting in a positive relationship between duration estimates and stimulus quantity. This assumption, however, seemed to be motivated mainly by empirical rather than theoretical considerations; in particular, by the results of studies on the filled-duration illusion indicating that brief temporal 
intervals (milliseconds to a few seconds) filled with stimuli are judged longer than equally long "empty" intervals.

Only a few studies (McClain, 1983; Predebon, in press; Vroon, 1970) have evaluated the relationship between prospective time estimates of moderately long intervals (i.e., greater than about $20 \mathrm{sec}$ ) and stimulus quantity. Vroon (1970) had subjects judge the relative durations of two 60 -sec-long intervals which differed in the number of presented auditory events (tones); the relationship was positive (i.e., the many-event interval was judged longer than the few-event interval) when subjects listened passively to the events, and negative (i.e., the few-event interval was judged longer) when subjects responded actively by classifying each event into one of two categories. A recent study (Predebon, in press), which used a methodology similar to Vroon's, confirmed a negative relationship when subjects actively responded to visual events (e.g., geometric patterns, oriented lines), but found either no relationship or a negative relationship under passive processing conditions. Vroon's finding of a positive relationship might be an artefact of the experimental instructions (Predebon, in press). If Vroon's subjects were not explicitly instructed to base their judgments on their subjective impressions, they may have judged the many-event interval as longer than the few-event interval, not because the two intervals were experienced different in duration, but because the many-event interval contained more events and, hence, from past experience, should be judged as longer. ${ }^{1}$ Such an explanation, however, cannot account for McClain's (1983) finding of a positive relationship inasmuch as her subjects were presented with only one interval for judgment. The interval events were words, and subjects performed a levels-of-processing task on each word. The number of words presented $(15,30$, or 45$)$ during the 120 -sec-long interval and the processing task was varied across subjects. Reproduction estimates were negatively related to the number of presented words when relatively deep levels of processing were required and positively related when shallow processing was required.

The positive relationship found in Vroon's passive and in McClain's shallow processing-task conditions suggests that the attention/distraction models of timing are incomplete, and that a different framework, such as Block's (1989) contextual change model or Hicks et al.'s (1977) model, which incorporates the distinction between active and passive processing, is needed to account for the interaction between stimulus quantity and the nature of the concurrent processing activity on prospective time estimates. In view of the implications of these findings for the attention/distraction model, the purpose of the two experiments described below was to further investigate the relationship between the number of presented stimuli and time estimates. The interval events were words, and subjects were required to (1) passively view the list of words, (2) graphemically encode the words (shallow processing), or (3) semantically encode the words (deep processing). The latter two tasks were identical to those used by McClain (1983). Subjective duration was assessed with the method of magnitude estimation in Experiment 1 and with the method of reproduction in Experiment 2.

\section{EXPERIMENT 1}

\section{Method}

Subjects. The subjects were 60 undergraduate students, all of whom participated in the experiment for course credit. Each student was tested individually.

Stimuli. There were two time intervals, each $60 \mathrm{sec}$ in duration, presented successively with a $5-\mathrm{sec}$ break between the intervals. Each interval consisted of the sequential presentation of a list of words appearing on the monitor of a Macintosh computer. One interval-the few-event interval-contained 14 words, and the other interval-the many-event interval-contained 28 words. Both intervals were delimited by the words START and END. The words were presented at rates of approximately one word every 4.2 and $2.1 \mathrm{sec}$ in the few- and manyevent intervals, respectively. Each word was presented for $1.5 \mathrm{sec}$. As in McClain's (1983) experiment, half of the words in each interval had "living" (e.g., teacher, wheat) and the other half had "nonliving" referents (e.g., stone, water). Half of the "living" and half of the "nonliving" words commenced with the letters A-L; the other halves commenced with the letters $M-Z$. The words were presented in a restricted random order. There were two sets of time intervals. For one set, the many-event interval was presented first and the few-event interval second $(\mathrm{M} / \mathrm{F})$; for the other set, the order was reversed $(\mathrm{F} / \mathrm{M})$. Different words were presented in the two sets of intervals.

Design and Procedure. The subjects were randomly assigned to one of three conditions of 20 subjects each: graphemic, semantic, and control. In the graphemic processing condition, the subjects classified each word as either commencing with a letter from the first (A-L) or the second $(\mathrm{M}-\mathrm{Z})$ half of the alphabet by pressing one of two designated keys on the computer keyboard, while in the semantic processing condition, the subjects classified each word as either "living" or "nonliving." The subjects in the control condition were instructed to passively look at the words. For each condition, half of the subjects were first presented with the $\mathrm{F} / \mathrm{M}$ sequence and then with the $\mathrm{M} / \mathrm{F}$ sequence; for the other half, the order of the two sequences was reversed.

All subjects were given practice on their designated task. The practice interval was $15 \mathrm{sec}$ long, and the words were presented at a rate intermediate between the rates of the few- and the many-event intervals. The subjects were then told that they would be presented with two intervals, and that they later would be asked to judge their relative durations. They were told not to count or otherwise mark time during the presentation of the word sequences. Immediately after the presentation of the first set of intervals, the subjects were given a response sheet for the magnitude-estimation time judgment. The response sheet contained an $80-\mathrm{mm}$-long reference line and a dot that was positioned directly below the left end of the line. The reference line represented the duration of the first interval. The subjects were required to draw a horizontal line, starting from the dot and extending rightward, so that the length of the response line relative to the length of the reference line represented their subjective impressions of the relative durations of the two intervals. The procedure was repeated for the second set of intervals. Thus, each subject made two magnitude estimates of subjective duration, one for the $M / F$ sequence and one for the $F / M$ sequence.

\section{Results and Discussion}

The omission and classification errors in the graphemic and semantic conditions were negligible $(<4 \%)$. The timeestimation judgments were converted to duration ratio $(R)$ scores by dividing the length of the reproduced line by the length $(80 \mathrm{~mm})$ of the reference line. Since the reference line designated the duration of the first-presented interval, ratios greater than 1.00 indicated that the first interval was judged shorter than the second interval, and ratios less than 1.00 indicated that the first interval was judged longer. The 
means and standard errors of the ratio scores for each of the six conditions of the experiment are shown in Table 1.

Experiment 1 constituted a 3 (group) $\times 2$ (sequence) factorial design with repeated measures on the sequence factor. The analysis of variance (ANOVA) performed on the data showed a significant effect of sequence $[F(1,57)$ $=19.62, p<.05]$, as well as a significant sequence $\times$ group interaction $[F(2,57)=4.57, p<.05]$. The effect of group was not significant $[F(2,57)=.02, p>.05]$. The nature of the interaction is evident in Table 1 ; the sequence factor differentially influenced time estimates in the graphemic and semantic conditions but not in the control condition. The results of the $t$ tests (two-tail) performed on the individual means showed that the means of the $\mathrm{M} / \mathrm{F}$ and $\mathrm{F} / \mathrm{M}$ ratios were significantly different from 1.00 for both the graphemic and semantic conditions (all $t \mathrm{~s}>2.15$ ) but not for the control condition $[t \mathrm{~s}(19)=1.45$ and 1.90 , respectively, $p<.05]$.

A positive relationship between time estimates and the number of presented stimuli is indicated by an estimation ratio $R<1.00$ for the $\mathrm{M} / \mathrm{F}$ sequence and a ratio $R>1.00$ for the $\mathrm{F} / \mathrm{M}$ condition; conversely, a negative relationship is indicated by an estimation $R>1.00$ and $R<1.00$ for the $\mathrm{M} / \mathrm{F}$ and $\mathrm{F} / \mathrm{M}$ sequences, respectively. For the control condition, the ratios are not significantly different from 1.00 , indicating that time estimates are unaffected by the number of presented stimuli. In contrast, both the graphemic and semantic tasks produced a negative relationship; the few-event interval was judged longer than the manyevent interval irrespective of whether the many-event interval was presented first $(M / F)$ or second $(F / M)$.

The negative relationship in the semantic task condition confirms previous findings (e.g., McClain, 1983; Predebon, in press), whereas the negative relationship in the graphemic task condition contradicts the positive relationship reported by McClain (1983). The latter inconsistency is especially puzzling, given the similarity of the interval events (words) and the identity of the graphemic task. Experiment 1 differed from McClain's experiment in other respects, however. For example, it used a withinsubjects rather than a between-subjects manipulation of the number of presented stimuli. The contribution of this procedural difference to the discrepant outcomes was evaluated by analyzing the first judgments of the subjects in the graphemic condition. The means and standard errors, in parentheses, of the time judgments of the $\mathrm{M} / \mathrm{F}$ sequence for the 10 subjects who were presented with the $M / F$ fol-

Table 1

Means and Standard Errors of Comparative Time Judgment Ratio Scores (First/Second Interval): Experiment 1

\begin{tabular}{ccrl}
\hline & Interval & & \\
Condition & Sequence & $M$ & $S E$ \\
\hline Graphemic & $\mathrm{M} / \mathrm{F}$ & 1.308 & .09 \\
& $\mathrm{~F} / \mathrm{M}$ & .843 & .07 \\
Semantic & $\mathrm{M} / \mathrm{F}$ & 1.313 & .10 \\
& $\mathrm{~F} / \mathrm{M}$ & .824 & .07 \\
Control & $\mathrm{M} / \mathrm{F}$ & 1.102 & .05 \\
& $\mathrm{~F} / \mathrm{M}$ & 1.201 & .04 \\
\hline
\end{tabular}

Note- $-\mathrm{M}$ and $\mathrm{F}$ refer to many- and few-event intervals, respectively. lowed by the F/M sequences and of the time judgments of the F/M sequence for the 10 subjects who were given the $\mathrm{F} / \mathrm{M}$ followed by the $\mathrm{M} / \mathrm{F}$ sequences were $1.42(.16)$ and $.87(.09)$, respectively; these values are similar to the group data. Alternatively, the inconsistency might reflect differences in the time judgment method (magnitude estimation vs. reproduction), although it is difficult to understand why such a difference should produce opposing outcomes for the graphemic task but virtually identical outcomes for the semantic task. Nevertheless, it seemed necessary to obtain converging evidence for the findings of Experiment 1. Experiment 2 therefore was similar to Experiment 1 , except that it presented single intervals for judgment and used the method of reproduction to measure subjective duration.

\section{EXPERIMENT 2}

\section{Method}

The two intervals were each $60 \mathrm{sec}$ long, and they were identical to the few- and many-event intervals of Experiment 1 . The 42 subjects, none of whom had participated in Experiment 1, were assigned randomly to one of three conditions of 14 subjects each: graphemic, semantic, and control. Each interval was presented individually for judgment. For half of the subjects in each condition, the many-event interval was presented first, while for the other half, the few-event interval was presented first. As in Experiment 1, the subjects were first given a practice session, after which they were informed that they would be required to judge the duration of the upcoming intervals. After the presentation of each interval, the subjects reproduced its duration. Subjects were instructed to say "Start," to sit quietly until an interval had elapsed which seemed subjectively equal to the interval in which the words had been presented, and then say "Stop." Each subject made two reproduction estimates, one for the few- and one for the many-event interval. In all other respects, Experiment 2 was identical to Experiment 1.

\section{Results}

The classification errors were negligible. The means and standard errors of the reproduced durations are shown in Table 2. The results of the mixed-design ANOVA showed significant effects of task $[F(2,39)=18.35, p<$ $.05]$ and stimulus number $[F(1,39)=31.81, p<.05]$, as well as a significant interaction between these variables $[F(2,39)=4.91, p<.05]$. Tests for simple effects indicated that the difference between the reproduced durations of the many- and the few-event intervals were significant for the graphemic and semantic task conditions $[F \mathrm{~s}(1,36)=13.49$ and 27.38 , respectively, $p<.05]$ but not for the control condition $[F(1,36)=.74, p>.05]$.

The results confirm the findings of Experiment 1; the many-event interval was experienced as being shorter than the few-event interval when active responding to the interval events was required (graphemic and semantic tasks), and was experienced as equal in duration to the many-event interval when active responding was not required (control condition). Additionally, the graphemic and semantic tasks yielded virtually identical outcomes.

\section{GENERAL DISCUSSION}

The results of the two experiments unequivocally demonstrate the independence of both magnitude time estimates and reproduced dura- 
Table 2

Means and Standard Errors of Reproduction Time Estimates (in Seconds): Experiment 2

\begin{tabular}{cccc}
\hline Condition & Interval & $M$ & $S E$ \\
\hline Graphemic & $\mathrm{M}$ & 31.4 & 2.40 \\
& $\mathrm{~F}$ & 41.0 & 2.89 \\
Semantic & $\mathrm{M}$ & 28.7 & 1.77 \\
& $\mathrm{~F}$ & 42.4 & 2.66 \\
Control & $\mathrm{M}$ & 51.4 & 3.05 \\
& $\mathrm{~F}$ & 53.7 & 2.65 \\
\hline
\end{tabular}

Note--M and $\mathrm{F}$ refer to many- and few-event intervals, respectively.

tions of moderately long intervals from the number of presented stimuli when active responding to the interval events is not required, and a negative relationship when active responding is required. Furthermore, the results indicate that the graphemic and semantic tasks have similar influences on time estimates.

The inconsistency between McClain's (1983) finding of a positive relationship and the negative relationship found here for an identical shallow processing (graphemic) task is difficult to resolve. Perhaps, and for whatever reason, the graphemic task was more difficult for the subjects tested in the experiments reported here than for her subjects, in which case the attentional demands of the graphemic and the semantic tasks of Experiments 1 and 2 were functionally equivalent. Nevertheless, if a positive relationship occurs under conditions of relatively low processing demands, as McClain assumed from Hicks's (Hicks et al., 1976; Hicks et al., 1977) model of prospective timing, then a positive relationship should be evident in the control conditions in which observers merely inspected the words; none was found. ${ }^{2}$

According to current interpretations of the attention/distraction models, neither the number of presented stimuli nor the nature of the concurrent nontemporal processing task in themselves convey temporal information. Rather, these factors influence subjective duration indirectly by diverting attention away from the temporal processor whose operation requires attentional resources that are shared with concurrent temporal processing activities. The present findings are entirely consistent with this framework. Irrespective of the stimulus presentation rate, passive viewing of the stimuli does not significantly divert attention away from time-in-passing and, hence, subjective duration is independent of the number of presented stimuli. When active responding to the interval events is required, attention is distracted away from timein-passing, and the degree of distraction plausibly increases with increases in the number of presented stimuli, resulting in a negative relationship between subjective duration and stimulus quantity.

The present findings, of course, do not exclude a positive relationship between stimulus quantity and subjective duration of brief temporal intervals $(<10 \mathrm{sec})$ under conditions of minimal processing of the interval events, as seems to be implied by the results of the filled-duration illusion studies (see Hicks et al., 1976). If this is the case, however, the results of the present investigation, together with other findings (Predebon, in press), suggest that the attentional models must be reformulated to account for the occurrence of a positive relationship between these variables for brief, but not for longer, interval durations.

Finally, a comment on the levels-of-processing manipulation is warranted. This technique is occasionally used in the time-estimation literature to assess the role of nontemporal processing demands on time estimation. The similarity of the effects found with the shallow and deep processing tasks suggest that little is gained by this manipulation. Indirect support for this claim is provided by Block (1992) and Bueno (1992). Block reported that three different depth-of-processing tasks (structural, rhyming, and category) yielded similar reproduction durations of a 180-sec-long interval, and Bueno failed to find a differential effect of two processing tasks (structural and semantic) on reproduction durations of an 80 -sec-long interval. In the context of the attention/ distraction model of timing, these findings, together with the results of
Experiments 1 and 2, suggest that the two different depth-of-processing tasks used here had similar distracting influences on the subject's ability to monitor time in passing, implying that they placed similar demands on attentional/processing resources.

\section{REFERENCES}

BLOCK, R. A. (1989). Experiencing and remembering time: Affordances, context, and cognition. In I. Levin \& D. Zakay (Eds.), Time and human cognition: A life-span perspective (pp. 333-363). Amsterdam: Elsevier, North-Holland.

BLOCK, R. A. (1992). Prospective and retrospective duration judgment: The role of information processing and memory. In F. Macar, V. Pouthas, \& W. J. Friedman (Eds.), Time, action and cognition (pp. 141152). Dordrecht: Klüwer.

BuENo, M. B. (1992). Testing models of time estimation. In F. Macar, V. Pouthas, \& W. J. Friedman (Eds.), Time, action and cognition (pp. 173-176). Dordrecht: Klüwer.

Hicks, R. E., Miller, G. W., Gaes, G., \& Bierman, K. (1977). Concurrent processing demands and the experience of time-in-passing. American Journal of Psychology, 90, 431-446.

Hicks, R. E., Miller, G. W., \& Kinsbourne, M. (1976). Prospective and retrospective judgments of time as a function of amount of information processed. American Journal of Psychology, 89, 719-730.

MCClaIN, L. (1983). Interval estimation: Effect of processing demands on prospective and retrospective reports. Perception \& Psychophysics, 34, 185-189.

Predebon, J. (1988). Retrospective time judgments and clock duration. Perceptual \& Motor Skills, 66, 19-24.

PREDEBON, J. (in press). The effects of active and passive processing of interval events on prospective and retrospective time estimates. Acta Psychologica.

Thomas, E. A. C., \& WeAver, W. B. (1975). Cognitive processing and time perception. Perception \& Psychophysics, 17, 363-367.

VROON, P. A. (1970). Effects of presented and processed information on duration experience. Acta Psychologica, 34, 115-121.

ZAKAY, D. (1993). Time estimation methods: Do they influence prospective duration estimates? Perception, 22, 91-101.

\section{NOTES}

1. Another possibility is that the positive relationship reflects a retrospective time component in Vroon's (1970) data. Each subject made two time judgments, one for the many-few and one for the few-many sequence. It is unclear from Vroon's description whether subjects were informed prior to the presentation of the first sequence that a duration judgment would be requested. If not, the first judgment involves a retrospective time judgment (i.e., subjects are unaware of the upcoming duration-judgment task). The second judgment, however, is likely to involve a prospective time judgment as subjects would now be alerted to the time task. As Vroon combined both the first and second time judgments, and since retrospective time estimates are positively related to stimulus quantity (e.g., McClain, 1983; Predebon, 1988), the positive relationship might be attributable to the first (retrospective) judgments in his data.

2 . To check on this possibility, an independent group of 14 subjects performed a shallow processing task-discrimination of upper- and lowercase words - that seemed less attention demanding than the graphemic task. The procedure was identical to Experiment 1. The same words were used, except that half were in uppercase and the other half were in lowercase. The mean ratios of the $\mathrm{M} / \mathrm{F}$ and the $\mathrm{F} / \mathrm{M}$ sequences were $1.175(S E=.08)$ and $.844(S E=.07)$, respectively. Both means are significantly different from 1.00 , indicating a negative relationship between time estimates and stimulus quantity.

(Manuscript received December 4, 1995; revision accepted for publication April 16, 1996.) 